\section{COLD DENSE MATTER}

$F^{\circ}$ OR his presidential address to the Section of Physics at the thirty-first Indian Science Congress, held at Delhi in January, Dr. D. S. Kothari took as his subject "Cold Dense Matter". Here the word 'cold' is used in a technical sense as meaning that any free electrons present constitute a degen. erate gas-the actual temperatures can range from planetary temperatures to those of the interiors of white dwarf stars. Dr. Kothari's address has now been published, and it gives us a welcome summary of the main features of temperature- and pressureionization of matter at high and low temperatures and at high and low densities.

The thermodynamic theory of ionization in gases, though first used in particular applications by Lindemann (now Lord Cherwell), was given its dominating importance in astrophysics by the work of Saha. Saha showed that the dissociation of atoms into ions and electrons is encouraged by reduction of pressure or increase of temperature. But, as Kothari points out, pressure variations are comparatively unimportant in influencing the degree of ionization unless the temperature is of a certain order of magnitude given by the relevant ionization potential ; for temperatures much lower or much higher, ionization will be either negligible or complete, whatever the pressure, and we therefore rightly describe Saha's ionization theory as the theory of temperature or thermal ionization. In his address, Kothari proceeds to discuss in some detail another kind of ionization, which he calls pressure ionization. This occurs in the case of relatively cold matter at high densities, when the atoms are pressed so tightly together that there is room neither for the outer electrons to be in bound orbits nor for them to be associated with particular ions. Kothari sums up the differences between thermal and pressure ionization in three compact propositions :

(i) Thermal ionization occurs in hot or nondegenerate matter; pressure ionization in cold or degenerate matter.

(ii) If, keeping temperature constant, the density be reduced, then the degree of ionization is increased for thermal ionization, and decreased for pressure ionization.

(iii) If, keeping density constant, the temperature be increased, the degree of ionization is increased in thermal ionization but remains practically unaffected in pressure ionization-unless the increase in temperature is so large that degeneracy is removed and pressure ionization passes into thermal ionization.

The discovery by Fermi and Dirac in 1926 of the correct form of quantum statistics to apply to gases had its repercussions on ionization theory : the early simple formulæ require modifications at large densities and low temperatures, where there sets in a 'degeneracy' which we have already mentioned. In addition, at very high temperatures further modifications are required, due to the need to use Einstein's modifications of Newtonian dynamics when the kinetic energy of thermal motion becomes comparable with the rest energy due to mass. The early part of Kothari's address contains a useful complete summary of the various discriminants which determine the different cases that arise at high or low densities or temperatures.

The later portions of his address are chiefly concerned with the applications of these general principles to the particular circumstances of the white dwarf stars and the planets. White dwarf stars were first recognized as configurations with degenerate cores by Sir Ralph Fowler. This theory has been considerably developed by Stoner and Chandrasekhar, and in his address Kothari rounds off these investigations, show. ing inter alia what had previously been assumed outright, namely, that for white dwarfs of mass exceeding a certain small critical mass, the stellar material is completely ionized. The application of the theory to bodies of planetary mass is a piece of original work of great beauty, due to Kothari himself. He shows that if we consider a series of 'cold' bodies of increasing mass, the radius increases to a maximum for a mass about one thousandth of that of the sun, and thereafter decreases ; the physical reason is that ultimately increase of mass so increases the central pressure that the atoms suffer such a degree of pressure ionization that they finally occupy a smaller total volume in spite of the increased mass. The value of the radius at the maximum is shown by Kothari to depend to some extent on the chemical composition of the material, and he infers that the observed mass/radius values for Jupiter and Saturn are consistent with their being formed largely of (dense) hydrogen, whereas the terrestrial planets (Mercury to Mars) lie nearer the iron curve. The address concludes with a closely reasoned account of the probable evolutionary history of a body of planetary mass, together with Kothari's own difficulties with his theory.

E. A. Mirne.

\section{MEDICAL EDUCATION IN INDIA}

$\mathrm{HOR}_{\mathrm{O}}$ his presidential address to the Section of 1 Medical and Veterinary Sciences at the thirty. first Indian Science Congress held at Delhi in January, Dr. K. V. Krishnan chose "Medical Education" as his subject. Progressive medical educationists in India are not; he said, satisfied with the existing medical educational institutions, and wish to reorganize them in the light of recent trends of Western medical thought. It is now more than a hundred years since the first medical college was established in India and it is time for a stock-taking. India and the U.S.S.R. are the only two countries in the world which still have a dual standard of medical education. and both have decided to abolish it. The U.S.S.R. has already done much to abolish the lower standard. Madras and the United Provinces have already abolished it. Elsewhere in India there are still the medical schools which turn out licentiates with a lower standard of education and the colleges which produce university graduates. In the very near future, Dr. Krishnan hopes, there will be only the medical colleges.

Most of these colleges are not, however, planned according to the principles laid down by Flexner in his "The Idea of a Modern University". India is still producing the "tradesman doctor"; and should go on, as Britain, the United States and the U.S.S.R. have done, to the production of medical men whose ideals are the service of the public, the prevention of disease and the maintenance of health, rather than the cure of illness after it has been atlowed to develop.

In the course of his address, Dr. Krishnan makes valuable suggestions for improvements along these lines. The libraries of the medical colleges should be extended and provided with foreign periodicals, textbooks should be produced which deal with Indian 